\title{
Results and Status from KamLAND-Zen
}

\section{Jonathan Ouellet*}

On behalf of the KamLAND-Zen Collaboration

Massachusetts Institute of Technology

E-mail: ouelletj@mit.edu

The KamLAND-Zen experiment is searching for the $0 v \beta \beta$ decay of ${ }^{136} \mathrm{Xe}$ using $320-380 \mathrm{~kg}$ of enriched Xe, mounted in the KamLAND detector in the Kamioka mine in Japan. In this talk, we report on the results of $504 \mathrm{~kg} \cdot \mathrm{yr}$ of data collected during the second phase of data taking, which followed a purification program of the liquid scintillator and Xe. Using this data, we set a limit on the $0 v \beta \beta$ half-life of $T_{1 / 2}^{0 v}\left({ }^{136} \mathrm{Xe}\right)>9.2 \times 10^{25} \mathrm{yr}$ at $90 \%$ C.L. Combined with the results of the first phase, this limit improves to $T_{1 / 2}^{0 v}\left({ }^{136} \mathrm{Xe}\right)>1.07 \times 10^{26} \mathrm{yr}$ at $90 \%$ C.L. Finally, we report on the current status of the next phase of the experiment, KamLAND-Zen 800.

38th International Conference on High Energy Physics 3-10 August 2016

Chicago, USA

${ }^{*}$ Speaker. 


\section{Introduction}

In the early 2000's, neutrino oscillation experiments showed conclusively that the neutrino is a massive particle $[1,2,3,4,5]$. Since then we have built a nearly complete picture of the oscillation parameters [6]. However, as of yet, we still do not know how to incorporate the neutrino mass into the Standard Model (SM). It is tempting to insert it analogously to all the other fermions in the SM, using the Higgs mechanism. However, this creates a question as to why the neutrino is some six orders of magnitude lighter than all of the other particle which get their mass through the same mechanism. The most elegant solution to this paradox, is to make the neutrino unique among the fermions in the SM and to give it a Majorana mass.

Majorana mass terms are typically low energy results of high energy physics, and thus the neutrino becomes light due to a seesaw mechanism. A Majorana mass also would imply that the neutrino is its own anti-particle, manifestly violating lepton number. This feature opens the possibility that the neutrino played a role in the production of the Baryon Asymmetry of the Universe and could help explain the abundance of matter in the Universe.

Neutrinoless Double-Beta $(0 v \beta \beta)$ Decay, $(Z, A) \rightarrow(Z+2, A)+2 e^{-}$, is an as-yet undiscovered process, which could only occur if the neutrino were a Majorana fermion. In recent years, the search for $0 v \beta \beta$ decay has garnered a lot of attention, with the largest experiments searching for the $0 v \beta \beta$ decay of ${ }^{76} \mathrm{Ge}[7],{ }^{130} \mathrm{Te}[8,9]$ and ${ }^{136} \mathrm{Xe}[10,11]$.

The $0 v \beta \beta$ decay rate is typically parameterized as

$$
\Gamma_{0 v}=\ln 2 G_{0 v}\left(Q_{\beta \beta}\right)\left|\mathscr{M}_{0 v}\right|^{2}\left|f_{0 v}\right|^{2} .
$$

Where $G_{0 v}\left(Q_{\beta \beta}\right)$ is a phase-space factor which depends on the full energy of the decay, as $\propto Q_{\beta \beta}^{5}$. $\mathscr{M}_{0 v}$ is the nuclear matrix element (NME), which parameterizing the overlap of the new nucleus $(Z+2, A)$, with the old, $(Z, A)$. All of the beyond the Standard Model physics is contained in the $f_{0 v}$ term. In the simplest model of light Majorana neutrino exchange, $f_{0 v}$ can be parameterized as

$$
\left|f_{0 v}\right|=\frac{\left\langle m_{\beta \beta}\right\rangle}{m_{e}}=\left|\frac{1}{m_{e}} \sum_{i} U_{e i}^{2} m_{i}\right| .
$$

Where $\left\langle m_{\beta \beta}\right\rangle$ is called the Effective Majorana Mass, the $U_{e i}$ are terms from the PMNS matrix, $m_{i}$ are the neutrino masses, and $m_{e}$ is the electron mass. Given the measured oscillation parameters, the allowed regions of $\left\langle m_{\beta \beta}\right\rangle$ are shown in Fig. 5.

The KamLAND-Zen experiment is a search for the $0 v \beta \beta$ decay of ${ }^{136} \mathrm{Xe}$ using a large liquid scintillator detector. The results described here are based on the work presented in [10].

\section{KamLAND-Zen Detector}

The KamLAND-Zen experiment is a search for the $0 v \beta \beta$ decay of ${ }^{136} \mathrm{Xe}$ mounted in the KamLAND detector in the Kamioka mine, Gifu prefecture, Japan. The mine provides an overburden of $1000 \mathrm{~m}$ of rock, which attenuates the muon flux by a factor of $\sim 10^{5}$ relative to the surface flux.

The KamLAND detector consists of a $18 \mathrm{~m}$ diameter sphere vessel containing a $13 \mathrm{~m}$ diameter outer balloon $(\mathrm{OB})$ filled with ultra-pure liquid scintillator (LS), suspended in a $1.8 \mathrm{~m}$ transparent 
layer of mineral oil that acts as a buffer. The walls of the spherical vessel are lined with 1,879 17" and 20" photomultiplier tubes (PMT), viewing the OB with a total coverage of $34 \%$. The spherical vessel is mounted in an 3,200 ton water Cherenkov detector that acts as both a muon veto and as a shield against radiation from the surrounding rock.

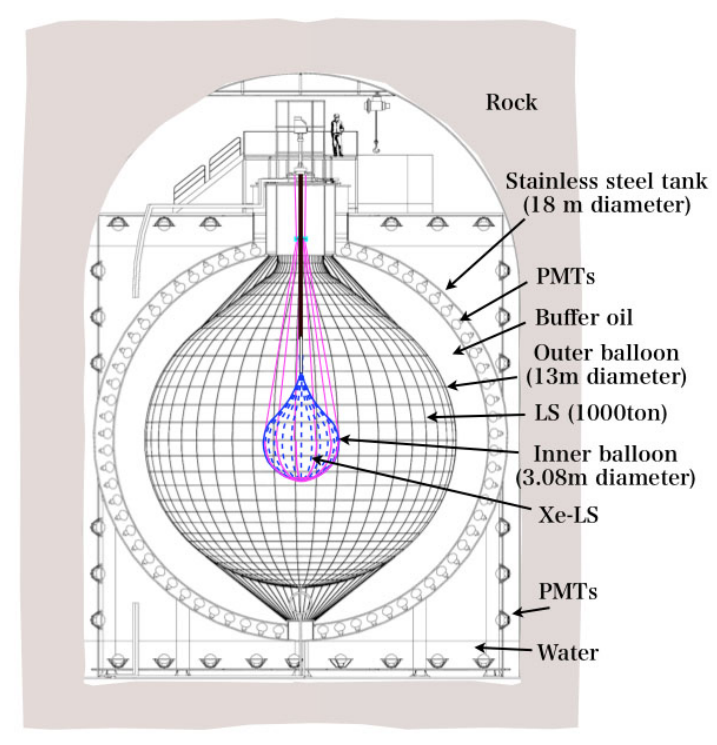

Figure 1: The KamLAND-Zen detector. The IB is a $3 \mathrm{~m}$ diameter balloon at the center of the KamLAND detector, viewed by 1,879 PMTs.
The KamLAND-Zen experiment focuses on a $3.08 \mathrm{~m}$ diameter inner balloon (IB), which has been installed at the center of the KamLAND detector (see Fig 1). This balloon is made from a $25 \mu \mathrm{m}$ thick nylon film, and contains a volume of LS loaded with $\mathrm{Xe}$ enriched to $91 \%{ }^{136} \mathrm{Xe}\left(Q_{\beta \beta}=\right.$ $2.458 \mathrm{MeV})$. The IB was produced in a class I clean room at Tohoku University in Sendai and transported to Kamioka in 2011.

KamLAND-Zen takes advantage of the KamLAND infrastructure which has been successfully studying reactor- $\bar{v}$ oscillations since 2002 [4]. The detector provides an ultra clean environment for a $0 v \beta \beta$ search with a well understood background and detector response. Since the IB only occupies $\sim 1.5 \%$ of the volume of the KamLAND detector, KamLAND-Zen can search for $0 v \beta \beta$ while the rest of the detector continues to pursue other physics goals, like supernova neutrino and geoneutrino searches [5].

\section{Results}

\subsection{Results of Phase I}

Phase I of data taking began in October 2011 with 320 kg of Xe and lasted until June 2012. This comprised an isotopic exposure of $89.5 \mathrm{~kg} \cdot \mathrm{yr}$ of ${ }^{136} \mathrm{Xe}$.

This initial data taking phase exhibited a significant and unexpected background contamination in the region of interest (ROI). The spectral shape of this background, and its time dependence, were consistent with the decay of ${ }^{110 \mathrm{~m}} \mathrm{Ag}$ ( $\left.\tau=360 \mathrm{~d}, Q=3.01 \mathrm{MeV}\right)$. We believe that this contamination was introduced when the IB was transported from Sendai, where it was produced, to Kamioka. During this time, it was exposed to the fallout from the 2011 Fukushima reactor accident. This interpretation is further supported by measuring the ratio of ${ }^{134} \mathrm{Cs}$ to ${ }^{137} \mathrm{Cs}$ on the IB, which matches that observed in soil samples around the reactor.

The background from ${ }^{110 \mathrm{~m}} \mathrm{Ag}$ creates a peak in the ROI, and thus significantly impacts the sensitivity to $0 v \beta \beta$. At the end of Phase I of data taking, we were able to set a limit on the $0 v \beta \beta$ half-life of ${ }^{136} \mathrm{Xe}$ at $T_{1 / 2}^{0 v}\left({ }^{136} \mathrm{Xe}\right)>1.9 \times 10^{25} \mathrm{yr}$ at $90 \%$ C.L. [12]. 


\subsection{Purification Campaign}

In June 2012, we concluded Phase I of data taking and began a purification campaign which lasted until October 2013. The first step of this process was to remove the Xe from the LS in the IB. During this time, we collected a Xe-depleted run of data and confirmed that while the ${ }^{136} \mathrm{Xe} 2 v \beta \beta$ rate fell precipitously, the contamination in the ROI remained. This was a nice demonstration of a source-on/off measurement that could be repeated to test a possible $0 v \beta \beta$ signal detection in the future.

We then passed the LS through three cycles of vacuum distillation; where each cycle corresponded to a single IB volume exchange. The Xe was purified through distillation, filtration and finally a heater zirconium getter, before being reintroduced back into the purified LS.

The results of this process were immediately apparent as a factor of $\sim 10$ drop in the ${ }^{110 \mathrm{~m}} \mathrm{Ag}$ rate at the center of the IB in the first 115 days of Phase II [13].

\subsection{Results of Phase II}

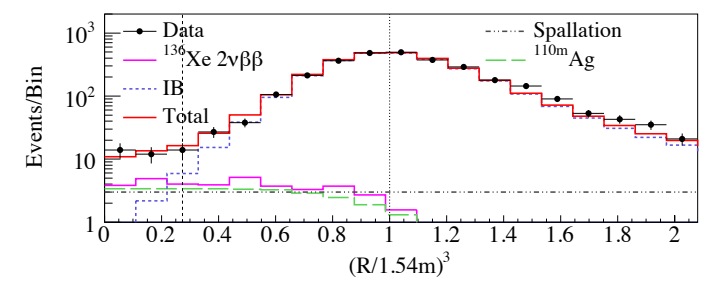

Figure 2: $R^{3}$ distribution of $0 v \beta \beta$ candidate events with $2.3<E<2.7 \mathrm{MeV}$. The dashed vertical line on the left corresponds to $R=1 \mathrm{~m}$.
Phase II of data taking began in November 2013, with a larger mass of $380 \mathrm{~kg}$ of enriched Xe. Data taking continued through October 2015, eventually collecting 534.5 days of livetime. The last month of data taking was dedicated to a calibration run, where sources were deployed along the central axis of the detector. The observed energy resolution was $\sigma / E=7.3 \% / \sqrt{E(\mathrm{MeV})}$. The observed position dependent energy and vertex bias was $<1 \%$ and $<1 \mathrm{~cm}$, respectively, for $|z|<1 \mathrm{~m}$ in the IB.

The fiducial volume is defined as $R<2 \mathrm{~m}$ and includes the full IB plus a $\sim 0.5 \mathrm{~m}$ buffer of LS around it. We identify ${ }^{214} \mathrm{Bi}^{214} \mathrm{Po}(\tau=237 \mu \mathrm{s})$ and ${ }^{212} \mathrm{Bi}^{212} \mathrm{Po}(\tau=0.43 \mu \mathrm{s})$ events using delayed coincidence and double pulse identification tags. We remove reactor $\bar{v}$ using the same tags used in previous analyses. We remove poorly reconstructed events, and add a 2 ms deadtime after muon events. After all cuts, the final exposure is $504 \mathrm{~kg} \cdot \mathrm{yr}$.

We tag the spallation product, ${ }^{10} \mathrm{C}$, using the triple-coincidence of muon-neutron- ${ }^{10} \mathrm{C}$, with the condition that the ${ }^{10} \mathrm{C}$ be within $1.6 \mathrm{~m}$ of the neutron and within $180 \mathrm{~s}$ of the muon event. The use of the new dead-time-free MoGURA electronics system has improved the efficiency of this to $(64 \pm 4) \%$ while maintaining a $93 \%$ signal efficiency. The other spallation product ${ }^{137} \mathrm{Xe}$ $\left(\beta^{-}, \tau=5.5 \mathrm{~min}, Q=4.17 \mathrm{MeV}\right)$ is produced mainly by neutron capture on ${ }^{136} \mathrm{Xe}$, and its rate is estimated from the known spallation neutron rate and the capture cross section.

Extracting and reintroducing the Xe loaded LS exposes it to ${ }^{222} \mathrm{Rn}(\tau=5.5 \mathrm{~d})$ which is pulled into the IB. This contamination decays quickly and provides a period of high rate data that we use for detector characterization. Most notably, the ${ }^{214} \mathrm{Bi}^{214} \mathrm{Po}$ delayed coincidence events in the LS can be tagged with $99.95 \%$ efficiency. We use these to study the efficiency of our vertex reconstruction algorithm and compare to Monte Carlo (MC). We also study the tail end of the ${ }^{214} \mathrm{Bi}$ 

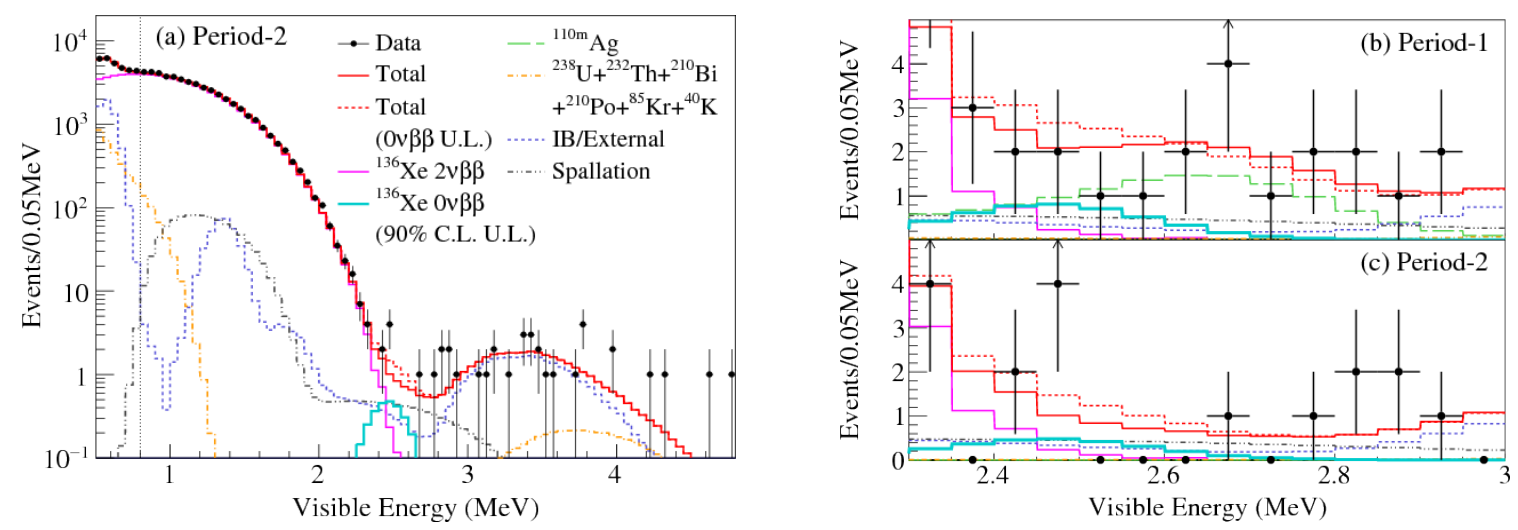

Figure 3: (a) Energy spectrum of $\beta \beta$ decay candidate events with $R<1 \mathrm{~m}$ from period-2 of Phase II of data taking with the best fit background spectra, $2 v \beta \beta$ spectrum and the $90 \%$ C.L. upper limit on the $0 v \beta \beta$ signal. (b),(c) Zoom of the measured energy spectrum and best-fit backgrounds between $2.3<E<3 \mathrm{MeV}$ and $R<1 \mathrm{~m}$ for period- 1 and period-2, respectively.

$\beta$-spectrum to search for any distortions of the high-energy tail. We saw no distortion and find that the tail is consistent with what we expect from a simple gaussian resolution.

Figure 2, shows the radial distribution of the observed events in the ROI. The dominant background is ${ }^{214} \mathrm{Bi}$ originating from the IB, but this falls off significantly at $R<1 \mathrm{~m}$ where ${ }^{110 \mathrm{~m}} \mathrm{Ag}$ is still present. Assuming the ${ }^{214} \mathrm{Bi}$ originates from the IB and assuming secular equilibrium, this contamination indicates a ${ }^{238} \mathrm{U}$ content in the film some 80 times larger than ICP-MS analysis suggests. We interpret this to mean that the majority of this background is introduced by "dust" stuck to the balloon surface.

We begin the analysis by splitting the fiducial volume into 40 equal volume bins. 20 bins in the upper hemisphere, 20 in the lower. Each bin covers the full $2 \pi$ in the azimuth, and fits the the radial dependence of the data. This analysis uses the full mass of the ${ }^{136} \mathrm{Xe}$. The energy range considered in this analysis goes from $0.5<E<4.8 \mathrm{MeV}$. Considering the apparent time dependence of the background rate, we further split Phase II of the data into two roughly equal periods: Period- 1 and Period-2 which comprise $270.7 \mathrm{~d}$ and $263.8 \mathrm{~d}$ of livetime, respectively. The background rate in the ROI appears to fall faster than one would expect from the natural decay of ${ }^{110 \mathrm{~m}} \mathrm{Ag}$. Though this is only a $\sim 2 \sigma$ effect, one possible explanation for it is ${ }^{110 \mathrm{~m}} \mathrm{Ag}$ sinking to the bottom of the IB and becomes lost in the ${ }^{214} \mathrm{Bi}$ background.

In the analysis, we assume several types of backgrounds: those free-floating in the Xe-loaded LS, those constrained to the balloon surface and those intrinsic to the whole detector (e.g. muon spallation). Many of these backgrounds are constrained from previous characterizations of the detector, and others can be constrained using tagged events. We perform a simultaneous fit of all free-floating parameters over all volume bins. Figure 3 a shows the full energy spectrum for the inner most volume bin $(R<1 \mathrm{~m})$ for Period-2 with the best fit background contributions. Figures $3 \mathrm{~b} \& 3 \mathrm{c}$ show a zoom of the same volume for Period-1 and 2, respectively. Figure 4 shows the radial and vertical distribution of events in the ROI for all of Phase II and indicates that a large fraction of the background is attributable to ${ }^{214} \mathrm{Bi}$ on the IB. Table 1 describes the best fit contributions the ROI background. 


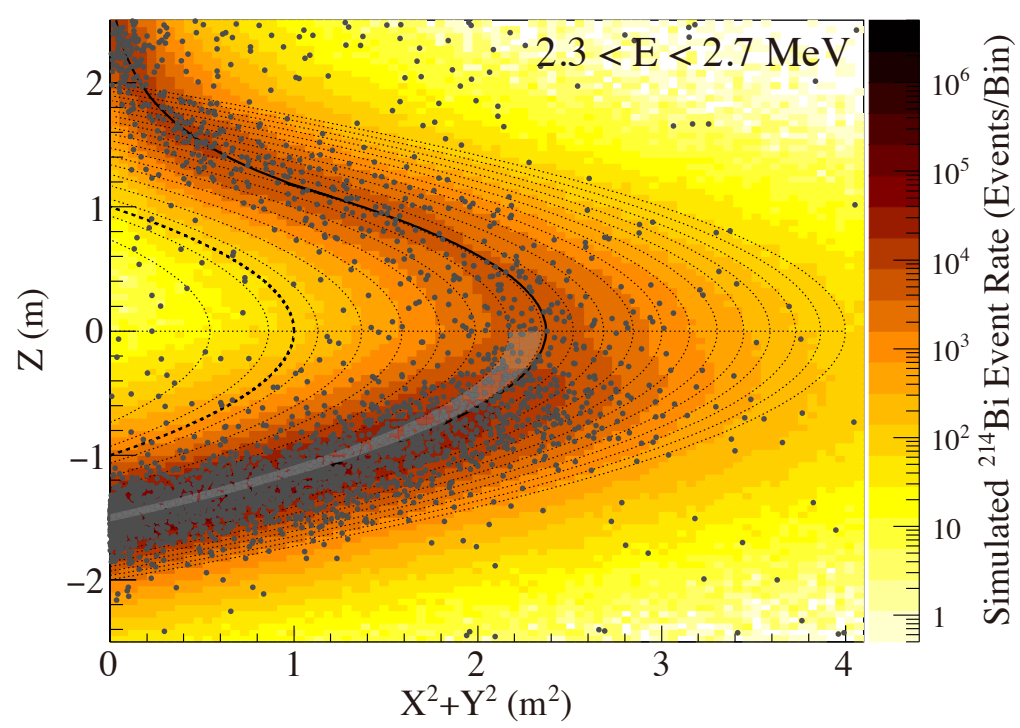

Figure 4: Distribution of Phase II events within the fiducial volume with energy $2.3<E<2.7 \mathrm{MeV}$. The gray points are the reconstructed event data. The color distribution is a MC simulation of ${ }^{214} \mathrm{Bi}^{2}{ }^{214} \mathrm{Po}$ cascade events originating from the IB surface. The thick black and dotted lines represent the IB surface and $R=1 \mathrm{~m}$. The thin dotted lines represent the surfaces of the 40 equal volume bins.

Our best fit $0 v \beta \beta$ component is consistent with no signal, and using Phase II data alone, we set a lower limit on the $0 v \beta \beta$ half-life of ${ }^{136} \mathrm{Xe}$ at $T_{1 / 2}^{0 v}\left({ }^{136} \mathrm{Xe}\right)>9.2 \times 10^{25} \mathrm{yr}$ at $90 \%$ C.L. Combining this with the limit from Phase I [12], we set a combined lower limit on the $0 v \beta \beta$ half-life of ${ }^{136} \mathrm{Xe}$ at $T_{1 / 2}^{0 v}\left({ }^{136} \mathrm{Xe}\right)>1.07 \times 10^{26} \mathrm{yr}$ at $90 \%$ C.L. Using the commonly used NME calculations, the most recent phase space calculation, and assuming $g_{A} \simeq 1.27$, this translates to a range of $90 \%$ C.L. upper limits on $\left\langle m_{\beta \beta}\right\rangle$ of $\left\langle m_{\beta \beta}\right\rangle<(61-165) \mathrm{meV}$. This result and a comparison to other isotopes is shown in Fig. 5.

We measure the $2 v \beta \beta$ half-life of ${ }^{136} \mathrm{Xe}$ using a modified fiducial volume to maximize the sensitivity to $2 v \beta \beta$ events of $R<1 \mathrm{~m}$. Performing a similar simultaneous fit to this reduced volume, yields a measurement of the $2 v \beta \beta$ half-life from Phase II data of $T_{1 / 2}^{2 v}\left({ }^{136} \mathrm{Xe}\right)=(2.21 \pm 0.02$ (stat. $) \pm 0.07$ (syst. $\left.)\right) \times 10^{21}$ yr. The systematic uncertainty on this measurement $(3.1 \%)$ is dominated by the uncertainty on the fiducial volume, consistent with the radial vertex bias of $1 \mathrm{~cm}$. This is consistent with the result of Phase I [14] and previous results from EXO-200 [15].

\section{Future Outlook}

Phase II of data taking concluded in October 2015, at which point we shut down the detector for an upgrade. In Spring 2016, we drained the outer detector and replaced the PMTs to improve veto efficiency.

We constructed a new IB in the clean room facility at Tohoku University, with improved cleanliness procedures. The new balloon will have twice the volume and will be filled with $750 \mathrm{~kg}$ of enriched Xe. The new balloon was transported from Sendai to Kamioka in specially designed vacuum packaging (to avoid contamination during transport), and as of August 2016 has 
Table 1: Summary of the number of observed events, and the estimated and best-fit backgrounds in the $0 v \beta \beta$ window $(2.3<E<2.7 \mathrm{MeV})$ within $R<1 \mathrm{~m}$ for period-1 and period-2.

\begin{tabular}{|c|c|c|c|c|}
\hline \multirow{3}{*}{$\begin{array}{l}\text { Observed Events } \\
\text { Background }\end{array}$} & \multicolumn{2}{|c|}{$\begin{array}{c}\text { Period-1 } \\
(270.7 \text { days }) \\
\end{array}$} & \multicolumn{2}{|c|}{$\begin{array}{c}\text { Period-2 } \\
\text { (263.8 days) }\end{array}$} \\
\hline & \multicolumn{2}{|c|}{22} & \multicolumn{2}{|c|}{11} \\
\hline & Estimated & Best-fit & Estimated & Best-fit \\
\hline${ }^{136} \mathrm{Xe} 2 v \beta \beta$ & - & 5.48 & - & 5.29 \\
\hline \multicolumn{5}{|c|}{ Residual (Xe-LS) } \\
\hline${ }^{214} \mathrm{Bi}\left({ }^{238} \mathrm{U}\right.$ series $)$ & $0.23 \pm 0.04$ & 0.25 & $0.028 \pm 0.005$ & 0.03 \\
\hline${ }^{208} \mathrm{Tl}\left({ }^{232} \mathrm{Th}\right.$ series $)$ & - & 0.001 & - & 0.001 \\
\hline${ }^{110 m} \mathrm{Ag}$ & - & 8.5 & - & 0.0 \\
\hline \multicolumn{5}{|c|}{ External (IB) } \\
\hline${ }^{214} \mathrm{Bi}\left({ }^{238} \mathrm{U}\right.$ series $)$ & - & 2.56 & - & 2.45 \\
\hline${ }^{208} \mathrm{Tl}\left({ }^{232} \mathrm{Th}\right.$ series $)$ & - & 0.02 & - & 0.03 \\
\hline${ }^{110 m} \mathrm{Ag}$ & - & 0.003 & - & 0.002 \\
\hline \multicolumn{5}{|c|}{ Spallation Products } \\
\hline${ }^{10} \mathrm{C}$ & $2.7 \pm 0.7$ & 3.3 & $2.6 \pm 0.7$ & 2.8 \\
\hline${ }^{6} \mathrm{He}$ & $0.07 \pm 0.18$ & 0.08 & $0.07 \pm 0.7$ & 0.08 \\
\hline${ }^{12} \mathrm{~B}$ & $0.15 \pm 0.04$ & 0.16 & $0.14 \pm 0.04$ & 0.15 \\
\hline${ }^{137} \mathrm{Xe}$ & $0.5 \pm 0.2$ & 0.5 & $0.5 \pm 0.2$ & 0.4 \\
\hline
\end{tabular}

arrived at Kamioka. Once installed and characterized, the new phase of data taking will be called KamLAND-Zen 800 , with an expected sensitivity down to $\left\langle m_{\beta \beta}\right\rangle \approx 40 \mathrm{meV}$ after 5 years of data taking.

In the future, we plan to upgrade the KamLAND detector to a new phase called KamLAND2 and a $0 v \beta \beta$ search called KamLAND2-Zen. This upgrade would include improving the energy resolution to $\sigma / E \approx 2 \%$ at the Q-value and further reductions to the background in the ROI. The goal is to be sensitive to $\left\langle m_{\beta \beta}\right\rangle=20 \mathrm{meV}$ and probe the full Inverted Hierarchy (IH). R\&D into these upgrades is ongoing and includes studies of LAB-based LS, high quantum-efficiency PMTs and light collectors, new LS purification techniques, new imaging systems for $\beta-\gamma$ separation, and scintillating films to reject events on the balloon surface. We are also exploring the possibility of a pressurized Xe-LS environment to increase the density of Xe.

\section{Acknowledgments}

The author would like to thank for the invitation and excellent hospitality throughout the conference. Thanks also go to KamLAND-Zen collaborators for providing information and comments. The KamLAND-Zen experiment is supported by JSPS KAKENHI Grants No. 21000001, No. 26104002 and No. 25220704; the World Premier International research Center Initiative, MEXT, Japan; Stichting FOM in the Netherland; and under the U.S. Department of Energy (DOE) Contract No. DE-AC02-05CH11231, as well as other DOE and NSF grants to individual institutions. 


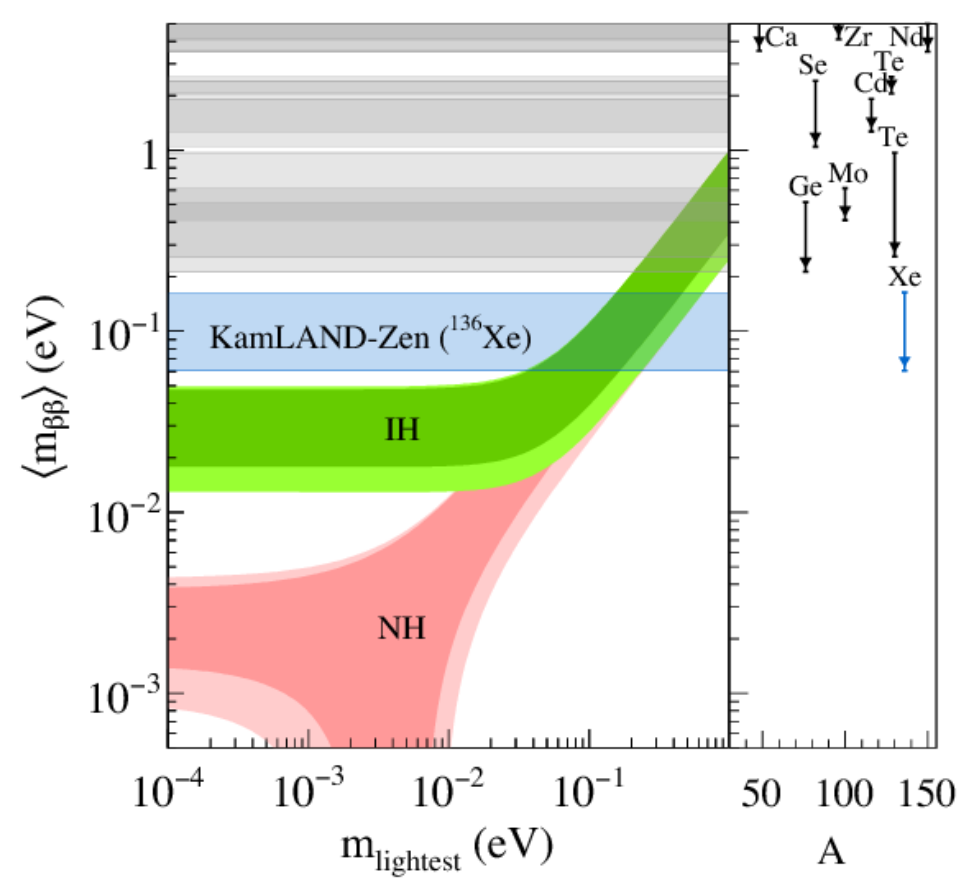

Figure 5: Limits on the effective Majorana neutrino mass $\left\langle m_{\beta \beta}\right\rangle$ from various isotopes. The allowed regions given best fit oscillation parameters are highlighted in red (NH) and green (IH). The lighter regions represent the $3 \sigma$ uncertainties on the oscillation parameters. Horizontal bands indicate $90 \%$ C.L. upper limits.

The Kamioka Mining and Smelting Company has provided service for activities in the mine. We acknowledge the support of NII for SINET4.

\section{References}

[1] S. Fukuda et al. Phys. Lett. B, 539:179-187, 2002.

[2] Q. R. Ahmad et al. Phys. Rev. Lett., 87:071301, Jul 2001.

[3] Q. R. Ahmad et al. Phys. Rev. Lett., 89:011301, 2002.

[4] K. Eguchi et al. Phys. Rev. Lett., 90:021802, 2003.

[5] A. Gando et al. Phys. Rev. D, 83:052002, 2011.

[6] K. A. Olive et al. (Particle Data Group), Chin. Phys C, 38, 0900012014.

[7] M. Agostini et al. Phys. Rev. Lett., 111(12):122503, 2013.

[8] K. Alfonso et al. Phys. Rev. Lett., 115:102502, 2015.

[9] C. Alduino et al. Phys. Rev. C, 93(4):045503, 2016.

[10] A. Gando et al. Phys. Rev. Lett., 117(8):082503, 2016.

[11] J.B. Albert et al. Nature, 510:229, 2014.

[12] A. Gando et al. Phys. Rev. Lett., 110(6):062502, 2013.

[13] K. Asakura et al. AIP Conference Proceedings, 1666:170003, 2015. 
[14] A. Gando et al. Phys. Rev. C, 86:021601, Aug 2012.

[15] J. B. Albert et al. Phys. Rev. C, 89:015502, Jan 2014. 